\title{
Movilidad y adaptación biomecánica: Una aproximación a la relación entre territorio y propiedades geométricas de la extremidad inferior en la población prehispánica de Gran Canaria.
}

\author{
Mobility and biomechanical adaptation: An approach to the relationship between territory and \\ geometric properties of the lower extremity in the pre-Hispanic population of Gran Canaria. \\ Jonathan Santana Cabrera ${ }^{(1)}$ Marco Moreno Benítez ${ }^{(2)}$ \\ G.I. Tarha. Departamento de Ciencias Históricas ${ }^{(1)}$ \\ C/ Pérez del Toro s/n. E-35003. Las Palmas de Gran Canaria, España. Teléfono: +34 928458913 / Fax: + 34 \\ 928452722. E-mail: jonathan.santana@ulpgc.es. \\ (1) G.I. Tarha. Departamento de Ciencias Históricas \\ (2) Tibicena, S.L. Gabinete de estudios patrimoniales.
}

\begin{abstract}
Resumen
La relación entre territorio y estructura ósea resulta importante si se quiere delimitar la influencia de este factor y establecer métodos de análisis más precisos en la investigación de la movilidad en poblaciones arqueológicas. Este artículo tiene como objetivo explorar la relación entre movilidad y territorio en la sociedad prehispánica de Gran Canaria (Islas Canarias, España). Se evalúan las propiedades geométricas de la extremidad inferior y su relación con la pendiente y el área de captación económica de los asentamientos vinculados a los asentamientos. Esta aportación constituye un modelo que sirve como referencia para el análisis de la movilidad y la influencia del territorio en poblaciones arqueológicas.
\end{abstract}

Palabras Claves: Arqueología, Bioarqueología, patrón de movilidad, restos humanos, SIG, osteometría. (5)

\begin{abstract}
The relationship between territory and bone structure is important to define the influence of this factor and establishing more precise methods of analysis in the investigation of mobility in archaeological populations. This article aims to explore the relationship between mobility and territory in pre-Hispanic society of Gran Canaria (Canary Islands, Spain). The geometric properties of the lower limb and its relationship with the slope and economic catchment area of settlements related to settlements are evaluated. This contribution is a model that serves as a reference for the analysis of mobility and influence of territory in archaeological populations.
\end{abstract}

Keywords: Archaeology, Bioarchaeology. Mobility pattern, human remains, GIS, ostemetric. 


\section{Introducción}

En Arqueología, la movilidad de los grupos humanos ha sido estudiada mediante diferentes perspectivas con el objeto de evaluar variados aspectos sociales. Las principales cuestiones se focalizan en el grado de sedentarización, el impacto de las estrategias de subsistencia, la organización socio-política, la territorialidad y el comercio [1][2].

Existen dos líneas principales para abordar la movilidad en las sociedades arqueológicas. La primera analiza la relación de la topografía del territorio con la ubicación de los asentamientos y las áreas de producción económica y político-simbólica [3]. La segunda vincula la actividad física de la movilidad con las características geométricas de los huesos de la extremidad inferior en humanos [4].

La primera línea de investigación aborda los criterios que explican el patrón de asentamiento y su relación con otros espacios, lo que deriva en una mejor comprensión de la naturaleza de sus prácticas socio-económicas. En este sentido, una de las principales herramientas para la relación de los asentamientos con su entorno más cercano lo constituye los estudios del área de captación económica (ACE). El ACE se fundamenta en la ley de los rendimientos decrecientes, cuyos principios básicos asocian directamente la función y localización de los asentamientos con respecto a la producción y a la ubicación de los productores [5]. De este modo, el espacio de explotación se define por la relación tiempo-distancia desde los contextos habitacionales a los territorios de aprovechamiento económico. Para las formaciones históricas con economía productora se propone como marco de referencia un área localizada a menos de una hora de camino desde el asentamiento a las zonas agrícolas, estableciéndose dicho tiempo por analogías etnográficas a partir de los estudios de Chisholm, donde la isócrona de 1 hora, suele fijar el límite del espacio productivo entre sociedades agrícolas [6].

La segunda línea de investigación se sustenta en varias aportaciones que han demostrado que existe una relación directa entre la morfología de estos huesos y el patrón de movilidad de los grupos humanos. Varios estudios que han analizado el impacto de ejercicios como la carrera o la escalada, han confirmado cómo la elevada contracción de los músculos isquiotibiales y los cuádriceps produce altas cargas de flexión en la mitad de la diáfisis de los fémures y de las tibias [4]. Como resultado, estas cargas mecánicas modifican la sección transversal de las diáfisis, que en un principio se caracterizan por tener una morfología circular, pero que progresivamente se transforman en una sección antero-posterior más alargada. De esta manera, las poblaciones arqueológicas con unos huesos más circulares representan un régimen de movilidad menor, y aquellas con una sección más alargada, se caracterizan por una movilidad más importante [4][7]. Esta capacidad de adaptación del esqueleto permite inferir cuestiones relativas al grado de sedentarización y al nivel de desplazamientos, pudiendo obtener datos importantes sobre las prácticas socio-económicas de las sociedades arqueológicas. Sin embargo, en esta adaptación también intervienen factores genéticos, climáticos, el tipo de terreno, la edad y el impacto de otras actividades físicas [4][7][8].

\section{Materiales y Métodos}

La muestra consta de 138 individuos (82 hombres y 56 mujeres) procedentes de diez necrópolis prehispánicas situadas en la costa de la isla de Gran Canaria (Tabla 1 y Fig. 1). Los restos humanos de estos cementerios están situados cronológicamente entre los siglos XI-XV y constituye una muestra poblacional representativa del último periodo de poblamiento prehispánico. Las necrópolis están representadas por conjuntos en fosas y cistas (Lomo Maspalomas, Juan Primo, Las Candelarias, Lomo Galeón, El Metropole), túmulos (El Agujero-La Guancha, Los Caserones) y cuevas funerarias (El Hormiguero, Lomo Los Gatos).

Tabla 1: Muestrasegúnsexo y necrópolis.

\begin{tabular}{|l|l|l|l|}
\hline Necrópolis & Asentamiento & Hombres & Mujeres \\
\hline $\begin{array}{l}\text { El Agujero- } \\
\text { La Guancha }\end{array}$ & Gáldar & 22 & 10 \\
\hline Juan Primo & Gáldar & 2 & 3 \\
\hline Maspalomas & Lomo Perera & 35 & 32 \\
\hline $\begin{array}{l}\text { Lomo } \\
\text { Galeón }\end{array}$ & El Pajar & 2 & 1 \\
\hline $\begin{array}{l}\text { Los } \\
\text { Caserones }\end{array}$ & Los Caserones & 2 & 3 \\
\hline El Metropole & El Metropole & 1 & 3 \\
\hline $\begin{array}{l}\text { El } \\
\text { Hormiguero }\end{array}$ & Guanchía & 10 & 1 \\
\hline El Risco & El Risco & 3 & \\
\hline $\begin{array}{l}\text { Las } \\
\text { Candelarias }\end{array}$ & Agaete & 2 & 1 \\
\hline $\begin{array}{l}\text { Lomo Los } \\
\text { Gatos }\end{array}$ & $\begin{array}{l}\text { Lomo Los } \\
\text { Gatos }\end{array}$ & 3 & 2 \\
\hline TOTAL & $\mathbf{1 3 8}$ & $\mathbf{8 2}$ & $\mathbf{5 6}$ \\
\hline
\end{tabular}

El sexo y la edad fueron estimados siguiendo los protocolos estándares. El sexo fue asignado a partir de 
los rasgos morfológicos del cráneo y la pelvis [9][10].La edad fue estimada mediante la conjunción de distintos métodos diagnósticos del esqueleto craneal (desgaste dental) y postcraneal (sínfisis púbica, faceta auricular, acetábulo, osificación del extremo esternal de las costillas) [9][11][12][13].

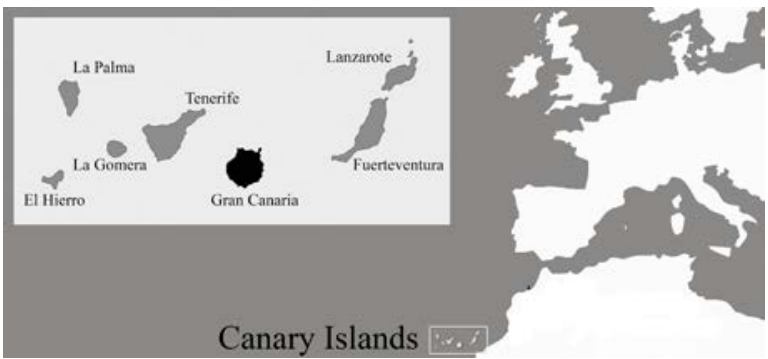

Figura 1. Situación de Gran Canaria en el archipiélago canario.

Los asentamientos vinculados a las diez necrópolis fueron seleccionados según criterios de proximidad y en base al conocimiento actual sobre el poblamiento prehispánico de la isla (Tabla 1). En la mayoría de estos espacios se tiene información del periodo de utilización, el cual, coincide plenamente con las dataciones radiocarbónicas de las necrópolis vinculadas [14]. El análisis territorial fue realizado a partir de la ubicación de estos asentamientos.

\subsection{Dimensiones métricas del esqueleto postcraneal}

El patrón de movilidad se investigó a partir de la caracterización morfológica de la sección transversal de los huesos largos de la extremidad inferior. Normalmente, la morfología de la sección transversal de los huesos de la extremidad inferior se estima a partir del índice de movilidad (lx/ly). Este parámetro describe la relación entre diámetro antero-posterior y diámetro latero-medial en las imágenes tomadas por TAC (tomografía axial computarizada) [4] [15]. No obstante, este método de representa una aproximación muy costosa en términos económicos y de accesibilidad. Como alternativa, otros parámetros métricos obtenidos a partir de las dimensiones exteriores de los huesos largos consiguen representar unos valores semejantes de la morfología de la sección transversal. Estos parámetros, además, han sido contrastados con el índice de movilidad (lx/ly) ofreciendo unos resultados casi idénticos [4][7][16]. En este artículo, se estudia la movilidad a partir del análisis de estos parámetros.

La muestra fue osteométricamente caracterizada tomando una serie de medidas en fémures y tibias según distintos manuales [16][17][18]. La toma de estas medidas se realizó con una tabla osteométrica SH-301, una cinta métrica de papel y un calibrador digital milimétrico de precisión Mitutoyo (0,05 mm). El patrón de movilidad fue calculado a partir de los índices de robustez, platimérico, pilastérico y cnémico de fémures y tibias [19] [7] [8]. Estos parámetros son capaces de aportar datos directos sobre la morfología de la sección transversal de los huesos de la extremidad inferior [7][15], y proporcionan una medida promedio de la fuerza y resistencia a la flexión del hueso [20].

\subsection{Análisis territorial}

Si bien existen metodologías que estudian las variables de la movilidad desde el estudio de las marcha y las vías de comunicación [2], el trabajo realizado fue un cálculo de la pendiente media del territorio anexo y el área de captación económica de los asentamientos vinculados a las necrópolis de procedencia de la muestra a través de un sistema de información geográfica de la isla de Gran Canaria (Tabla 1). Con este fin se utilizó un modelo Digital de Elevaciones (MDE) proporcionado por Cartográfica de Canarias SA (GRAFCAN). Este modelo a escala 1:5.000 (cinco metros de pixel) es lo suficientemente preciso para los requerimientos técnicos necesitados. La existencia de este MDE nos liberó de la realización de un ráster insular. Partiendo de aquel se generó una superficie de fricción teniendo como base el algoritmo de A. Uriarte [21], siendo esta fórmula la matematización de los datos de Gilman y Thornes[22]. Se trata de una fórmula anisótropica, es decir, que contabiliza de forma similar los costes tanto de ascenso como de descenso de una pendiente. Por ello, para el modelo posterior de coste hemos utilizado un algoritmo (pathdistance) que ponderaría parcialmente dicho movimiento tanto en subidas como en bajada.

A partir de esa base cartográfica se elaboraron las propuestas de territorios de explotación , usando para ello la realización de diferentes polígonos isocronos (basados en una aproximación más realista, ya que considera en su realización la distancia y el esfuerzo realizado) a tramos de 15 minutos (Figura 2). Posteriormente, y a partir del alcance espacial de cada isocrona, se cuantificó el número de metros cuadrados, y la pendiente media de cada una.

\subsection{Análisis estadístico}

Los diferentes índices examinados se calcularon con el programa informático Microsoft Excel (versión 2007). Las diferencias entre índices y grupos poblacionales fueron analizadas con el test noparamétrico de Kruskall-Wallis. Este test también fue utilizado para avaluar las diferencias en el área y pendiente de las áreas de captación económica de cada 
asentamiento/necrópolis. El tratamiento estadístico de los datos se realizó con el paquete informático SPSS para Windows (Versión 15.0).

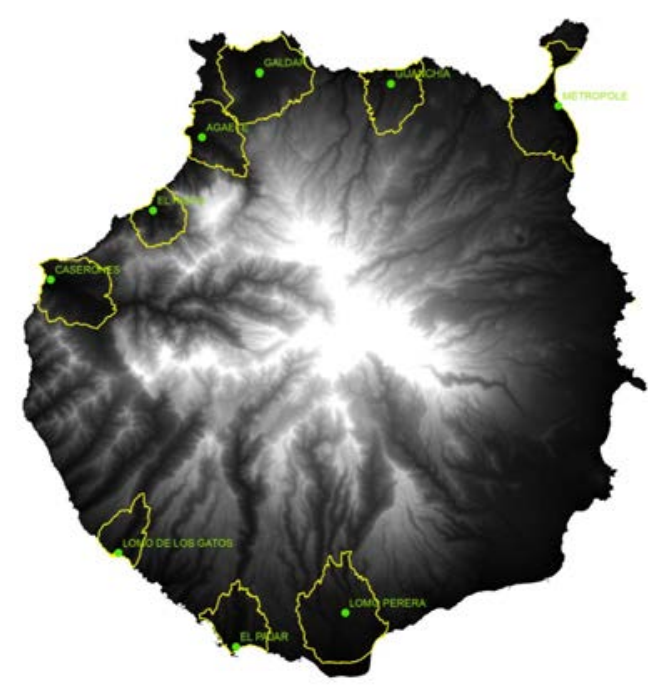

Figura2: ACE a los 60 minutos de los asentamientos asociados a las necrópolis de procedencia de la muestra.

\section{Resultados}

Los resultados principales del conjunto de índices para toda la muestra se exponen en la Tabla 2. El índice platimérico de la población de Gran Canaria puede definirse como hiperplatimérico según la clasificación de Olivier [17]. Este marcador informa sobre el grado de aplastamiento del extremo superior del fémur, circunstancia que se ha relacionado con un desarrollo importante del músculo bíceps femoral debido a un régimen de actividad importante de las piernas [23][7].

Tabla 2: Resultados generales de los índices de la extremidad inferior en el conjunto de la muestra.

\begin{tabular}{|l|l|r|r|r|r|r|}
\hline & & Platimérico & Pilstérico & Cnémico & \multicolumn{1}{c|}{$\begin{array}{c}\text { Robustez } \\
\text { fémur }\end{array}$} & $\begin{array}{c}\text { Robustez } \\
\text { tibia }\end{array}$ \\
\hline \multirow{7}{*}{ Hombre } & Media & $\mathbf{8 1 , 2 4 5 1}$ & $\mathbf{1 1 7 , 9 7 7 1}$ & $\mathbf{6 6 , 9 6 9 0}$ & $\mathbf{2 0 , 0 3 3 5}$ & $\mathbf{2 2 , 4 7 4 7}$ \\
\cline { 2 - 7 } & $\mathrm{N}$ & 67 & 47 & 70 & 53 & 56 \\
\cline { 2 - 7 } & $\begin{array}{l}\text { Desv. } \\
\text { típ. }\end{array}$ & 9,50081 & 8,76062 & 5,60809 & 1,53025 & 1,57257 \\
\hline \multirow{3}{*}{ Mujer } & Media & $\mathbf{8 3 , 4 8 8 1}$ & $\mathbf{1 1 3 , 8 8 0 0}$ & $\mathbf{6 5 , 4 0 7 3}$ & $\mathbf{1 8 , 6 8 5 9}$ & $\mathbf{2 0 , 9 7 0 5}$ \\
\cline { 2 - 7 } & $\mathrm{N}$ & 51 & 24 & 56 & 19 & 35 \\
\cline { 2 - 7 } & $\begin{array}{l}\text { Desv. } \\
\text { típ. }\end{array}$ & 18,13362 & 16,85591 & 9,16466 & 1,66811 & 1,54429 \\
\hline \multirow{3}{*}{ Total } & Media & $\mathbf{8 1 , 9 9 3 4}$ & $\mathbf{1 1 6 , 3 5 9 1}$ & $\mathbf{6 6 , 3 1 6 8}$ & $\mathbf{1 9 , 6 7 7 4}$ & $\mathbf{2 1 , 9 2 8 9}$ \\
\cline { 2 - 7 } & $\mathrm{N}$ & 121 & 73 & 128 & 73 & 93 \\
\cline { 2 - 7 } & $\begin{array}{l}\text { Desv. } \\
\text { típ. }\end{array}$ & 13,79010 & 12,07558 & 7,36334 & 1,65518 & 1,71533 \\
\hline
\end{tabular}

Los valores del índice pilástrico son elevados. Este parámetro responde al grado de relieve de la línea áspera del fémur, que cuando es muy pronunciada, dibuja una especie de "columna" en la sección transversal del hueso. Su desarrollo se relaciona con un patrón significativo de esfuerzos físicos en la extremidad inferior [24]. Los datos recopilados de la muestra de Gran Canaria señala que hombres y mujeres tenían como valores medios una sección transversal definida como "pilastra media” según la nomenclatura de Olivier [17],aunque más bajos para el grupo femenino. El índice cnémico también fue 3. más elevado en el conjunto de los hombres.

Los valores de los índices de robustez también fueron elevados con cifras superiores en el conjunto masculino.

Los resultados osteométricos de la población prehispánica examinada indican un patrón de movilidad reducido semejante a los observados en poblaciones sedentarias con una economía productora consolidada (Tablas 3 y 4) [4] [7] [19][15].

Tabla 3: Índices del fémur en distintas poblaciones arqueológicas con modos de vida diferentes. Sólo HOMBRES. (Datos propios y tomados de Bridges et al., 2000; Wanneret al., 2007 y Pomeroy y Zakrzewsky, 2009).

\begin{tabular}{|c|c|c|c|c|}
\hline Hombres & $\begin{array}{c}\text { Platimérico } \\
\text { derecho }\end{array}$ & $\begin{array}{c}\text { Platimérico } \\
\text { izquierdo }\end{array}$ & $\begin{array}{c}\text { Pilastérico } \\
\text { derecho }\end{array}$ & $\begin{array}{c}\text { Pilastérico } \\
\text { izquierdo }\end{array}$ \\
\hline Gran Canaria & 79,7 & 80,5 & 117,6 & 116,5 \\
\hline MW & 75 & 76 & 111 & 117 \\
\hline ELW & 75 & 75 & 117 & 117 \\
\hline LLW & 74 & 73 & 112 & 114 \\
\hline MISS & 75 & 73 & 118 & 115 \\
\hline Clásico Maya & 85,1 & 82,5 & 108,4 & 107,2 \\
\hline Écija & 86,03 & 86,32 & 113,33 & 109,96 \\
\hline $\begin{array}{c}\text { Great } \\
\text { Chesterford }\end{array}$ & 88,4 & 86,34 & 106,16 & 102,96 \\
\hline
\end{tabular}

Para evaluar la relación entre territorio y parámetros métricos de la extremidad inferior se examinaron los índices pilastérico, platimérico, cnémico y de robustez de fémur y tibia según la necrópolis de origen (Tabla 5). En El Hormiguero los valores platiméricos fueron más bajos que en el resto de poblaciones. En cambio, en contextos poco montañosos, como en Maspalomas, las cifras del índice platimérico fueron más altas. En cuanto al pilastérico, destaca la serie de El Agujero-La Guancha con medidas superiores al resto de las necrópolis. Por su parte, es la población de El Risco la que presenta un índice pilastérico más bajo. Para el parámetro cnémico de la tibia, la mayor puntuación la registraron Juan Primo, Lomo Los Gatos y Los Caserones, mientras que la más baja fue para El Metropole. En este sentido, la población de Gran 
Canaria tiene unos valores comprendidos en la mesonecmia y euricnemia, lo que indicaría poco aplanamiento transversal. La robustez del fémur fue más elevada en la serie de Lomo Galeón y El Risco y menos desarrollada en las series de Los Caserones y Maspalomas. Finalmente, los valores de la robustez de la tibia fueron nuevamente mayores en Lomo Galeón y menores en Juan Primo.

Tabla 4: Índices del fémur en distintas poblaciones arqueológicas con modos de vida diferentes. Sólo MUJERES. (Datos propios y tomados de Bridges et al., 2000; Wanneret al., 2007 y Pomeroy y Zakrzewsky, 2009).

\begin{tabular}{|c|c|c|c|c|}
\hline Mujeres & $\begin{array}{c}\text { Platimérico } \\
\text { derecho }\end{array}$ & $\begin{array}{c}\text { Platimérico } \\
\text { izquierdo }\end{array}$ & $\begin{array}{c}\text { Pilastérico } \\
\text { derecho }\end{array}$ & $\begin{array}{c}\text { Pilastérico } \\
\text { izquierdo }\end{array}$ \\
\hline $\begin{array}{c}\text { Gran } \\
\text { Canaria }\end{array}$ & 80,8543 & 84,83 & 115,4971 & 109,8382 \\
\hline MW & 73 & 73 & 106 & 106 \\
\hline ELW & 73 & 73 & 106 & 105 \\
\hline LLW & 73 & 72 & 104 & 106 \\
\hline MISS & 73 & 73 & 109 & 112 \\
\hline $\begin{array}{c}\text { Clásico } \\
\text { Maya }\end{array}$ & 81,5 & 78,5 & 98,5 & 98,1 \\
\hline Écija & 83,59 & 82,69 & 104,51 & 102,12 \\
\hline $\begin{array}{c}\text { Great } \\
\text { Chesterford }\end{array}$ & 81,4 & 82,19 & 99,1 & 98,36 \\
\hline
\end{tabular}

El área de captación económica (ACE) de los diez asentamientos/necrópolis examinados no presentó variaciones estadísticas entre asentamientos en las isócronas a 15, 30 y 45 minutos. Únicamente en el ACE a 60 minutos se observó una diferencia estadística entre asentamientos (Área: x2=241,129; $\mathrm{p}<0,001$. Pendiente: $x 2=241,034 ; \mathrm{p}<0,001$ ) (Figuras 3 y 4). Estos datos sugieren que es a partir de los 60 minutos de marcha cuando la orografía del territorio constituye un factor influyente en el patrón de movilidad.
Tabla 5. Valores medios de los índices según la necrópolis de origen.

\begin{tabular}{|c|c|c|c|c|c|}
\hline Necrópolis & Platimérico & Pilastérico & Cnémico & $\begin{array}{c}\text { Robustez } \\
\text { Fémur }\end{array}$ & $\begin{array}{c}\text { Robustez } \\
\text { Tibia }\end{array}$ \\
\hline $\begin{array}{c}\text { El Agujero-La } \\
\text { Guancha }\end{array}$ & 78,93 & 119,28 & 64,4299 & 19,9957 & 22,2716 \\
\hline Juan Primo & 77,14 & 113,45 & 72,9079 & 19,5063 & 19,8166 \\
\hline Maspalomas & 83,57 & 112,14 & 66,7296 & 18,9415 & 21,8846 \\
\hline Lomo Galeón & 77,69 & 110,88 & 66,5540 & 22,4772 & 23,0974 \\
\hline Los Caserones & 79,88 & 114,26 & 71,4916 & 17,9325 & 21,9640 \\
\hline El Metropole & & 111,91 & 59,4190 & 19,6947 & 20,0575 \\
\hline $\begin{array}{c}\text { El } \\
\text { Hormiguero }\end{array}$ & 74,89 & 115,09 & 69,9919 & 20,1916 & 21,7009 \\
\hline El Risco & 80,71 & 104,08 & 65,7057 & 20,6019 & 23,4807 \\
\hline $\begin{array}{c}\text { Las } \\
\text { Candelarias }\end{array}$ & 76,16 & 112,74 & 66,5997 & 19,5430 & 23,2646 \\
\hline $\begin{array}{c}\text { Lomo Los } \\
\text { Gatos }\end{array}$ & - & $\boldsymbol{p}<\mathbf{0 , 0 0 5}$ & - & $\boldsymbol{p}<\mathbf{0 , 0 0 5}$ & - \\
\hline $\begin{array}{c}\text { Pvalores } \\
\text { hombres }\end{array}$ & - & - & - & - & - \\
\hline $\begin{array}{c}\text { Pvalores } \\
\text { mujeres }\end{array}$ & - & 72,0979 & & 22,1341 \\
\hline
\end{tabular}

Asimismo, los datos indican que el patrón de movilidad de los antiguos canarios se vincula con el conocido para formaciones históricas de economía productora, con un nivel de desplazamientos que resulta homogéneo entre asentamientos a menos de 60 minutos de marcha [21].

Las diferencias estadísticas de los asentamientos también se observaron en las dimensiones métricas de los restos óseos (Tabla 5). Por ejemplo, los fémures y tibias de las necrópolis de El Agujero-La Guancha y Maspalomas resultaron ser las menos robustas de todo el conjunto analizado, con un ACE mayor que el resto de contextos arqueológicos a 60 minutos, y con las pendientes más suaves de toda la serie. Por su parte, El Risco y Lomo Los Gatos revelaban los fémures más robustos y el territorio inmediato con las pendientes más pronunciadas, lo que se tradujo en un “área de captación” de extensión menor. Esta correspondencia viene a corroborar la influencia del territorio en la musculatura y el patrón de movilidad de la población prehispánica. Son precisamente los asentamientos con un territorio más escarpado aquellos que tienen una población con mayor robustez en la extremidad inferior.

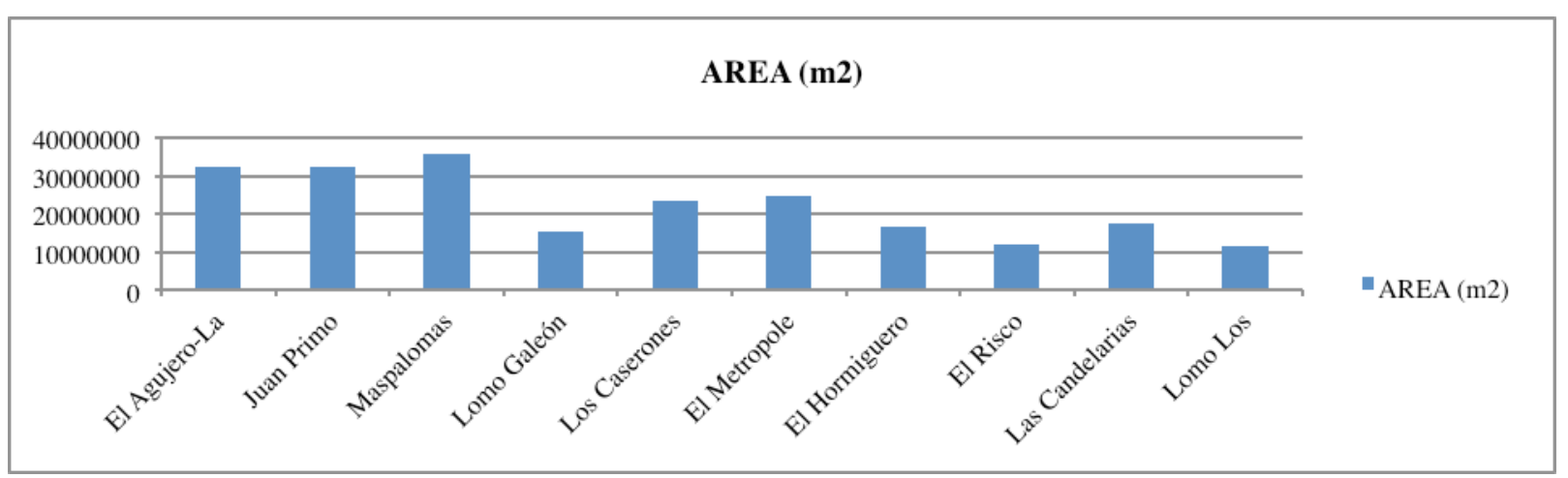

Figura 3: Área del ACE de cada asentamiento a 60 minutos. 


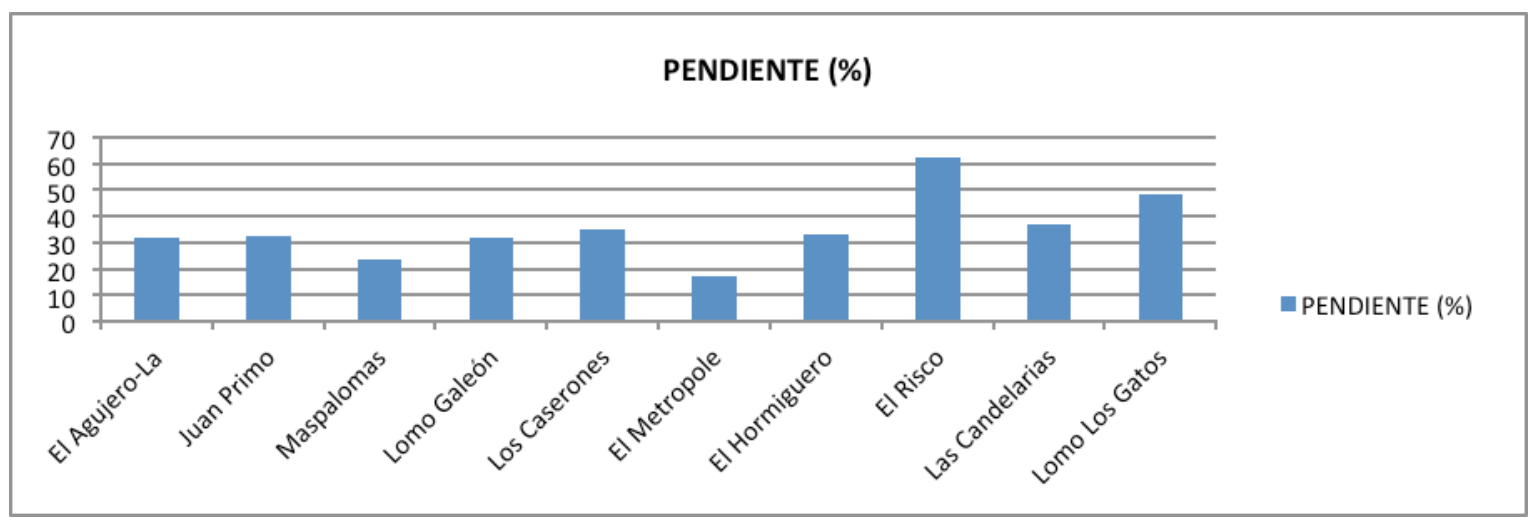

Figura 4: Pendiente del ACE de cada asentamiento a 60 minutos.

Pero la orografía no explica toda la variabilidad del fenómeno. Por ejemplo, a la serie de Juan Primo se le asignó el mismo asentamiento que a la necrópolis de El Agujero-La Guancha, el núcleo urbano de Gáldar. Sin embargo, los resultados indican que los individuos de Juan Primo, con unos fémures menos circulares, tenían un patrón de movilidad mayor que los de El Agujero-La Guancha. Es aquí donde podrían influir otros aspectos ligados a la organización social de la producción y al modo de acceso a los espacios productivos y a los productos.

\section{Conclusiones}

La aproximación expuesta en este artículo pone de manifiesto la posibilidad de integrar dos líneas de investigación dispares en el estudio de la movilidad de las poblaciones arqueológicas. Los resultados observados para la población prehispánica de Gran Canaria sugieren que la orografía del territorio jugó un papel importante en la adaptación biomecánica de los individuos. Aun así, los datos también sugieren que otros factores, quizás de tipo social, intervienen en la configuración estructural de las extremidades inferiores como resultado de la marcha. Esta línea de trabajo precisa de nuevas poblaciones para profundizar en la complejidad del fenómeno y corroborar el comportamiento observado en esta primera aproximación.

\section{Referencias}

[1]Kelly R. L., 1992. Mobility/sedentism: concepts, archaeological measures, and effects. Annual Review of Anthropology, 21: 43-66.

[2] Murrieta-Flores P., 2012 Entendiendo la movilidad humana mediante tecnologías espaciales: el papel de las áreas naturales de tránsito en el Suroeste de la Península Ibérica durante la Prehistoria reciente, Trabajos de Prehistoria, 69 (1): 103-122.

[3] García San Juan L., 2005. Introducción al Reconocimiento y Análisis Arqueológico del Territorio. Ariel Prehistoria.Barcelona.

[4] Ruff C.B., 2008. Biomechanical analyses of archeological human skeletons. En: Katzenberg M.A., Saunders S.R. Biological anthropology of the human skeleton. Wiley-Liss. New York. pp. 183-206.

[5] Vicent García J.M., 1991. Fundamentos teóricometodológicos para un programa de investigación arqueo-geográfica. En: López García, P.: El cambio cultural del IV al II milenios A.C. en la comarca de Murcia. Consejo superior de investigaciones científicas. Madrid: 31-117.

[6] Chisholm M.D.I., 1962 Rural Settlement and land use: an essay in location. Transaction Books. Londres.

[7]Wescott D., 2005. Population variation in femur subtrochanteric shape. J Forensic Sci, 50: 286-293.

[8] Bridges P., Blitz J., Solano M., 2000 .Changes in long bone diaphyseal strength with horticultural intensification in west central Illinois. American Journal of Physical Anthropology 112:217-238.

[9] Buikstra J.E., Ubelaker D., (Eds.), 1994. Standards for data collection from human skeletal remains. Arkansas Archaeological Survey Research Series, Number 44.Fayetteville. 
[10] Bruzek J., 2002. A method for visual determination of sex, using the human hip bone. Am J PhysAnthropol117: 157-168.

[11] Brothwell D., 1987. Desenterrando huesos. La excavación, tratamiento y estudio de restos del esqueleto humano. Fondo de la Cultura Económica.Madrid.

[12] Schmitt A., 2005. Une nouvelle méthode pour estimerl'âgeaudécès des adultes à partir de la surface sacro-pelvienneiliaque. Bull mem soc anthropol Paris 17: 89-101.

[13] Rissech C., Estabrook G., Cunha E., Malgosa A., 2007. Estimation of age-at-deathforadult males using the acetabulum, applied to four western European populations. J ForensicSci 52 (4): 774-778.

[14] Santana Cabrera J., 2011. El trabajo fosilizado: patrón cotidiano de actividad física y organización social del trabajo en la Gran Canaria prehispánica. Tesis doctoral. Universidad de Las Palmas de Gran Canaria.

[15] Pomeroy E.,Zakrzewski S.R., 2009. Sexual dimorphism in diaphyseal cross-sectional shape in the Medieval Muslim population of Écija, Spain and Anglo-Saxon Great Chesterford, UK. International Journal of Osteoarchaeology 19: 50-65.

[16]Larsen C.S., 1997. Bioarchaeology. Interpreting behavior from the human skeleton. Cambirdge University Press.

[17] Olivier G., 1960. Pratique anthropologique. Vigotfrères. Paris.

[18] Campillo D., Subirá M. E., 2004. Antropología Física para arqueólogos. Colección Ariel Prehistoria.

Ariel. Barcelona.

[19] Wanner I.S., Sosa T.S., Alt K.W., Blos V.T., 2007. Lifestyle, Occupation, and Whole Bone Morphology of the Pre-Hispanic Maya Coastal Population from Xcambo Yucatan, Mexico. International Journal of Osteoarchaeology 268: 253268.

[20] Cole T., 1994. Size and shape of the femur and tibia in northern Plains Indians. En: Owsley, D., Jantz, R. Skeletal biology in the Great Plains: migration, warfare, health, and subsistence. Smithsonian Institution Press. Washington, DC. pp. 219-234.

[21] Uriarte González A., (2005): Arqueología del Paisaje y Sistemas de Información Geográfica: una aplicación en el estudio de las sociedades protohistóricas de la cuenca del Guadiana Menor (Andalucía oriental), Bronce Final y Edad del Hierro en la Península Ibérica (A. Blanco, A.C., Cancelo, A. Esparza, Eds.), Colección Aquila fuente 96, Ediciones Universidad de Salamanca. Salamanca. pp. 603-621.
[22] Gilman A., Thornes J.B., 1985. Land-use and Prehistory in southeast of Spain. Allen \&Unwin. Londres.

[23]Malgosa A., 1992. La población talaiòtica de Mallorca. Intitut d'Estudis Catalans. Barcelona.

[24]Kennedy K.A.R., 1989. Skeletal markers of occupational stress. En: Iscan M., Kennedy, K.A.R., Reconstruction of Life from the skeleton. Wiley-Liss, Nueva York. pp. 129-160. 